\title{
Nonlinear Hierarchical Control Allocation for Vehicle Yaw Stabilization and Rollover Prevention
}

\author{
Matthäus B. Alberding*,**, Johannes Tjønnås*, Tor A. Johansen* \\ *Department of Engineering Cybernetics, Norwegian University of Science and Technology, Trondheim, Norway \\ ** Institute for Systems Theory and Automatic Control, University of Stuttgart, Germany
}

\begin{abstract}
In ambition to minimize potential interferences between yaw stabilization and rollover prevention of an automotive vehicle, this work presents a new approach to integrate both objectives. It introduces rollover prevention in form of a nonlinear constraint on the control allocation of a yaw stabilizing controller, yielding a hierarchical allocation problem. A suitable algorithm in form of a dynamic update law addressing this problem is derived. Its implementation is computationally efficient and suitable for low cost automotive electronic control units. The proposed rollover constraint design does not require any sensory equipment in addition to the yaw stabilizing algorithm. Actuation is conducted by differential braking, while an extension to further actuators is possible. The method is validated using an industrial multi-body vehicle simulator.
\end{abstract}

Index Terms-Automotive; Constrained control; Safety critical systems

\section{INTRODUCTION}

Control systems for active safety are among the major advances in automotive technology over the past decades. Anti-lock Braking Systems (ABS) to improve braking performance and Electronic Stability Programs (ESP) designed to control the yaw motion and prevent skidding, are standard in modern road vehicles. However, vehicles with a high center of gravity like Light Trucks and Vans (LTVs) or Sports Utility Vehicles (SUVs), show under extreme maneuvering a notable vulnerability towards rollover accidents. To address this problem, a new generation of active safety systems is required.

Various strategies for rollover prevention have been proposed [4], [9], [11]. In order to address yaw stabilization and rollover prevention simultaneously, the relationship between these problems has to be taken into account. The central problem in this context are cases where the demands of these two tasks conflict and cannot be satisfied concurrently. It is clear that in case of imminent rollover threat, rollover prevention must be granted priority over yaw stabilization. However, as long as the risk to roll over is small, yaw stabilization has to be preferred. Due to its modular structure and existing studies in vehicle dynamics control [9], [14], [17], [18], control allocation-based approaches are especially suited to address this problem. Such a control scheme has been developed in [12], [13]. There, rollover prevention is delegated to a threshold-switched controller. Mapping this virtual control to the actual control input is prioritized over a yaw stabilizing virtual control by a respectively chosen weighting matrix in an allocation algorithm based on active set programming methods.

In the present work, we take a new approach to the problem, where we emphasize on the task to grant yaw stabilization as much freedom as possible, provided that rollover is prevented. Therefore, the idea of a high-level roll controller is abandoned completely, and instead rollover prevention introduced as constraint on the allocation of yaw control. This represents a natural approach to rollover, which can rather be seen as a constraint on the vehicle's motion than a classical control problem dealing with stabilization around a defined reference. Its major advantage compared to a controller-based approach lies in the consideration of the full set of solutions preventing rollover, thus interfering with yaw control only if unavoidable. A central aspect will be the development of a suitable allocation algorithm that addresses the hierarchical problem resulting from this approach. The proposed predictive design of the rollover constraint does not require any sensory equipment in addition to the yaw stabilizing algorithm. We consider differential braking as available actuators, but it is possible to extend the results of this work to further actuators like active steering or active suspension.

\section{MODELING}

Consideration of a control allocation strategy divides vehicle modeling in two parts: a high-level model with a virtual input of forces and moments, and an associated actuator/effector model, providing the relationship between this virtual input and the actual control input. The high-level dynamics describe the chassis motion, while the actuator model is mainly related to tire-road interaction and the braking system. An overview over all model variables and parameters is given in Table I and illustrated in Fig. 1-2.

In order to describe the chassis dynamics, let us employ a nonlinear two-track model based on [10] with roll dynamics [12]:

$$
\begin{aligned}
& {\left[\begin{array}{c}
\dot{v} \\
\dot{\beta} \\
\ddot{\psi}
\end{array}\right]=-\left[\begin{array}{c}
0 \\
\dot{\psi} \\
0
\end{array}\right]+\left[\begin{array}{ccc}
\frac{1}{m} \cos \beta & \frac{1}{m} \sin \beta & 0 \\
-\frac{1}{m v} \sin \beta & \frac{1}{m v} \cos \beta & 0 \\
0 & 0 & \frac{1}{J_{z}}
\end{array}\right]\left[\begin{array}{c}
F_{x T} \\
F_{y T} \\
M_{z T}
\end{array}\right]} \\
& {\left[\begin{array}{c}
\dot{\varphi} \\
\ddot{\varphi}
\end{array}\right]=\left[\begin{array}{cc}
0 & 1 \\
\frac{m g h-C_{\varphi}}{J_{x}} & -\frac{K_{\varphi}}{J_{x}}
\end{array}\right]\left[\begin{array}{c}
\varphi \\
\dot{\varphi}
\end{array}\right]-\left[\begin{array}{c}
0 \\
\frac{h}{J_{x}}
\end{array}\right] F_{y T}}
\end{aligned}
$$

Note that direct couplings between yaw and roll accelerations are neglected.

It has already been mentioned that this work considers actuators related to a differential braking system, thus, the actual control input consists of braking pressures $p_{b, i}$. But due to the utilization of an ABS with a controller able to track a commanded longitudinal wheel slip, we relate the actuator model to wheel slip. According to [10], the virtual control vector can be obtained from the individual wheel forces:

$$
\left[\begin{array}{c}
F_{x T} \\
F_{y T}
\end{array}\right]:=\sum_{i=1}^{4}\left[\begin{array}{cc}
\cos \delta_{w, i} & -\sin \delta_{w, i} \\
\sin \delta_{w, i} & \cos \delta_{w, i}
\end{array}\right]\left[\begin{array}{l}
F_{x, i} \\
F_{y, i}
\end{array}\right]
$$




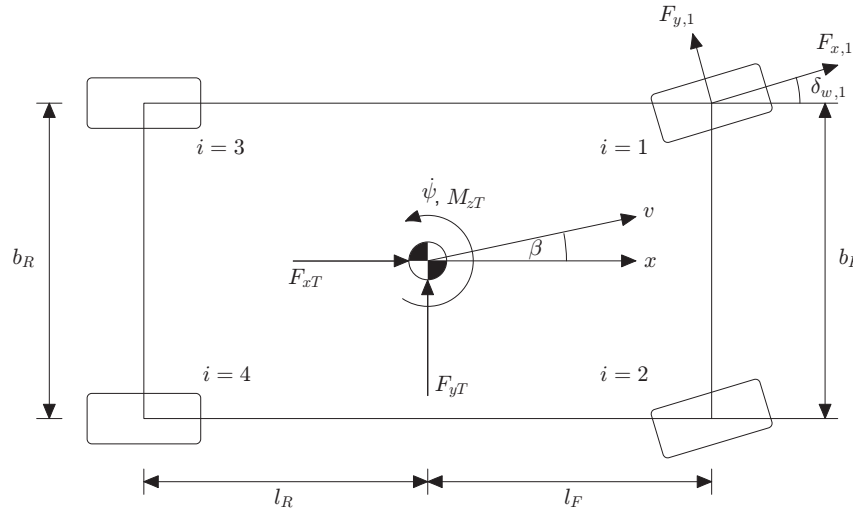

Fig. 1. Vehicle schematic in the horizontal plane

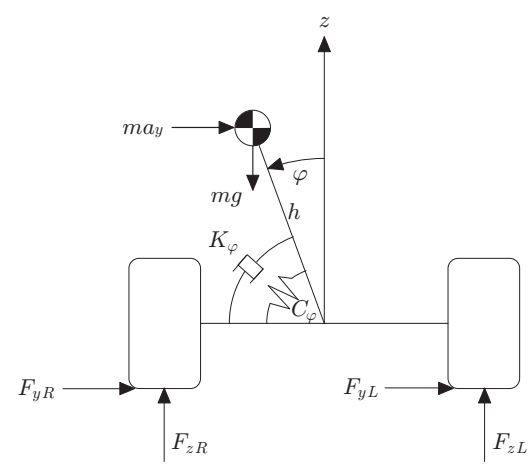

Fig. 2. Vehicle schematic in the vertical plane, showing suspension modeled as torsional spring and damper

and

$$
\begin{aligned}
M_{z T} & :=\sum_{i=1}^{4}\left[\begin{array}{l}
b_{i} \\
l_{i}
\end{array}\right]^{\mathrm{T}}\left[\begin{array}{cc}
\cos \delta_{w, i} & -\sin \delta_{w, i} \\
\sin \delta_{w, i} & \cos \delta_{w, i}
\end{array}\right]\left[\begin{array}{c}
F_{x, i} \\
F_{y, i}
\end{array}\right] \\
b_{1,2} & :=\mp \frac{b_{F}}{2}, b_{3,4}:=\mp \frac{b_{R}}{2}, l_{1,2}:=l_{F}, l_{3,4}:=l_{R} .
\end{aligned}
$$

For a relationship between individual wheel forces and longitudinal wheel slip, we use a tire-road friction model of the form

$$
\begin{aligned}
& F_{x, i}:=-F_{z, i} \mu_{x, i}\left(\lambda_{x, i}, \alpha_{i}, \mu_{H, i}\right) \\
& F_{y, i}:=-F_{z, i} \mu_{y, i}\left(\lambda_{x, i}, \alpha_{i}, \mu_{H, i}\right) .
\end{aligned}
$$

The employed control allocation approach does not rely strongly on the detailed structure of the friction model, we assume it satisfies the following qualitative properties:

(i) $\frac{\partial \mu_{x, i}}{\partial \lambda_{x, i}}>0$ for small longitudinal slips.

(ii) $\frac{\partial \mu_{x, i}}{\partial \mu_{H, i}}>0$ and $\frac{\partial \mu_{y, i}}{\partial \mu_{H, i}}>0$ for sufficiently large longitudinal slip.

(iii) The first order partial derivatives of the friction parameters with respect to the maximum tire-road friction dominate the higher order partial derivatives.

These assumptions are typically satisfied (for a given range of longitudinal wheel slip) in general friction models such as Pacejka's magic formula and Burckhardt's approach [2]. A more detailed discussion of the friction model assumptions and properties can be found in [17].

\begin{tabular}{|ll|}
\hline$i$ & Subscript for each wheel, see Fig. 1 \\
$\beta$ & Absolute vehicle velocity at center of gravity \\
$\psi$ & Vehicle side slip angle \\
$\varphi$ & Yaw angle \\
$F_{x T}, F_{y T}$ & Roll angle \\
$M_{z T}$ & Total longitudinal/lateral wheel force \\
$F_{x, i}, F_{y, i}, F_{z, i}$ & Total torque around the yaw axis \\
$p_{b, i}$ & Longitudinal/lateral/normal wheel force \\
$\delta_{w, i}$ & Braking pressure \\
$\delta_{s}$ & Wheel turn angle \\
$\alpha_{i}$ & Steering wheel angle \\
$\lambda_{x, i}$ & Tire side slip angle, tan $\alpha_{i}:=-\frac{v_{y, i}}{v_{x, i}}$ \\
$v_{i}, v_{x, i}, v_{y, i}, \omega_{i}$ & Wheel absolute/longitudinal/lateral/angular velocity \\
$\mu_{H, i}, \mu_{x, i}, \mu_{y}, i$ & Maximum/longitudinal/lateral tire-road friction \\
$m$ & coefficient \\
$J_{x}, J_{y}, J_{z}$ & Total vehicle mass \\
$h$ & Moment of inertia around roll/pitch/yaw axis \\
$h_{r}$ & Height of center of gravity above roll axis \\
$C_{\varphi}, K_{\varphi}$ & Height of roll axis above ground \\
$l_{F}, l_{R}$ & Total roll stiffness/damping \\
$b_{F}, b_{R}$ & Distance of front/rear axle to center of gravity \\
$R$ & Track width front/rear \\
& Wheel radius \\
\hline
\end{tabular}

TABLE I

MODEL VARIABLES AND PARAMETERS

\section{CONTROL ARCHITECTURE}

The architecture of the presented vehicle dynamics control is depicted in Fig. 3. It consists of following elements:

a) Nonlinear Observer: In order to generate estimates of vehicle velocity and side slip angle based on yaw rate, wheel speed, acceleration and steering angle measurements, a nonlinear observer based on [7] is utilized, also providing an estimate for the maximum tire-road friction parameters $\hat{\mu}_{H, i}$. All other states and variables used in the algorithm are viewed as sampled measurements.

b) High-Level Yaw Stabilizing Controller: As presented in [14], based on the yaw dynamics in (1) a virtual control law $M_{z T, c}$ in form of a PI-controller is designed such that the origin of the error dynamics described by $\dot{\tilde{\psi}}=\dot{\psi}-\dot{\psi}_{\text {ref }}$ is uniformly globally asymptotically stable for $M_{z T}=M_{z T, c}$. The yaw rate reference $\dot{\psi}_{\text {ref }}$ is generated on the basis of the driver's steering input by consideration of the steady-state of the vehicle side-slip dynamics (1).

c) Low-Level Braking Controller: Utilizing a standard production Anti-lock Braking System (ABS) accepting commanded longitudinal wheel forces $F_{x, i}^{\text {des }}$, founding on [17] control laws for these forces are defined such that for any smooth reference $\lambda_{x, i}^{\text {des }}$, the longitudinal wheel slip $\lambda_{x, i}$ will track $\lambda_{x, i}^{\text {des }}$ asymptotically.

d) Control Allocation: The main contribution of this work is the development of a nonlinear control allocation algorithm that addresses the hierarchical allocation problem of mapping a yaw stabilizing virtual input under a constraint representing rollover prevention. It defines dynamic update laws for the desired longitudinal wheel slip $\lambda_{x, i}^{\text {des }}$, tracked by the low level braking controller, such that the yaw stabilizing virtual control $M_{z T, c}$ is tracked under consideration of a constraint designed to prevent rollover. Further, minimum braking and actuator constraints are incorporated in form of an instantaneous cost function. 


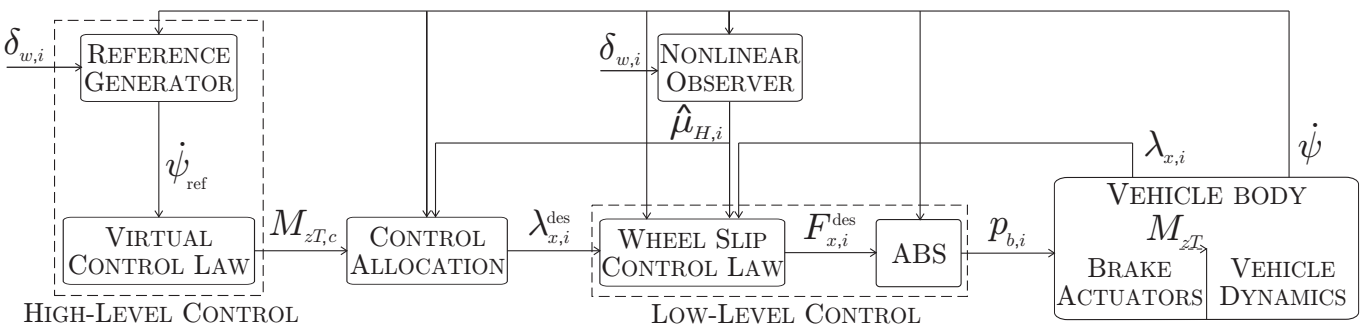

Fig. 3. Control architecture

\section{CONTROL ALLOCATION}

To allow a nonlinear formulation of the (yet to be defined) constraint representing rollover prevention, nonlinear allocation methods have to be considered. The presented control allocation in form of a dynamic update law is based on the nonlinear optimizing allocation algorithm developed in $[8]^{1}$. This addresses an over-actuated nonlinear system of the form

$$
\begin{aligned}
\dot{x} & =f(t, x, \tau) \\
\tau & =h(t, x, u),
\end{aligned}
$$

where $t \geq 0$ denotes time, $x \in \mathbb{R}^{n}$ the state vector, $u \in \mathbb{R}^{r}$ the control input vector and $\tau \in \mathbb{R}^{p}$ the vector of virtual controls. Further, $\tau_{c} \in \mathbb{R}^{p}$ represents the virtual control generated by a high-level controller and $J(t, x, u)$ an instantaneous cost function. Then, the basic control allocation problem of mapping a requested virtual input under minimum cost to the control input, can be formulated by the nonlinear static minimization problem

$$
\min _{u} J(t, x, u) \quad \text { s.t. } \quad \tau_{c}-h(t, x, u)=0 .
$$

For the given application, control input $u=\lambda_{x, i}^{\text {des }}$ refers to the desired longitudinal wheel slip, $\tau_{c}=M_{z T, c}$ to the yaw stabilizing virtual control and $h(t, x, u)=M_{z T}$ to actuator model (4)-(5). The states of the high-level dynamics (1)-(2) are treated as known time-varying signals. Hence, mapping of the virtual control addresses yaw stabilization, while the cost function is defined such that minimum braking and actuator constraints $u_{\min } \leq u \leq u_{\max }$ are incorporated:

$$
J(u):=u^{\mathrm{T}} H u-w_{u} \sum_{i=1}^{4} \ln \left(u_{i}-u_{\min }\right)-w_{u} \sum_{i=1}^{4} \ln \left(-u_{i}+u_{\max }\right),
$$

where $H=H^{\mathrm{T}}>0$ and $w_{u}>0$.

The central thought of this work is to introduce rollover prevention by granting a constraint $C_{e}(t, x, u)=0$ priority over exact mapping of the virtual control. This means to reformulate the allocation problem by

$$
\begin{aligned}
u & =\arg \min _{u \in \Omega} J(t, x, u) \\
\Omega & =\arg \min _{u}\left\|\tau_{c}-h(t, x, u)\right\| \text { s.t. } C_{e}(t, x, u)=0,
\end{aligned}
$$

where $\Omega(t, x)$ is the possibly uncountable set of all solutions $u$ to $(9 \mathrm{~b})$. Before addressing this problem, let us derive a suitable rollover constraint $C_{e}(t, x, u)=0$. In order to be compatible to the proposed allocation algorithm, $C_{e}(t, x, u)$ has to satisfy the following constraint controllability-like

\footnotetext{
${ }^{1}$ For further considerations with emphasis on extensions and assumption relaxation incorporating adaptation and actuator dynamics, the interested reader may refer to [15], [16]
}

condition [1]:

Assumption 4.1: There exists a constant $\mu>0$ such that

$$
\frac{\partial C_{e}}{\partial u}(t, x, u) \frac{\partial C_{e}^{\mathrm{T}}}{\partial u}(t, x, u) \geq \mu I_{s}
$$

\section{A. Rollover Constraint Design}

There are various approaches to determine indices that quantify how close the vehicle is to begin rolling over [3], [5], [9], [11]. In this work, we employ an energy-based approach derived in [9]. We define the rollover threat index

$$
\Delta(\varphi, \dot{\varphi}):=\frac{E(\varphi, \dot{\varphi})}{E_{\text {crit }}}
$$

where

$$
\begin{aligned}
& E(\varphi, \dot{\varphi})=\frac{1}{2} C_{\varphi} \varphi^{2}-m g h(1-\cos \varphi)+\frac{1}{2}\left(J_{x}+m h^{2}\right) \dot{\varphi}^{2} \\
& E_{\text {crit }}=E\left(\varphi_{\text {crit }}, 0\right), \quad \varphi_{\text {crit }}:=\frac{m g}{C_{\varphi}}\left(\frac{1}{2} b_{F}-\mu_{H} h_{r}\right) .
\end{aligned}
$$

This means, we relate the potential and kinetic energy of the roll system to a critical energy for which steady-state wheel lift-off is possible.

In order to be able to satisfy Assumption 4.1, dependency on the control input is necessary. This can be introduced by considering a predicted value after a time step $T_{p}$

$$
\Delta_{\text {pred }}\left(\varphi, \dot{\varphi}, \delta_{w, i}, u\right):=\Delta(\varphi, \dot{\varphi})+T_{p} \dot{\Delta}\left(\varphi, \dot{\varphi}, \delta_{w, i}, u\right),
$$

where

$$
\begin{aligned}
\dot{\Delta}\left(\varphi, \dot{\varphi}, \delta_{w, i}, u\right)= & \frac{1}{E_{\text {crit }}}\left(C_{\varphi} \varphi \dot{\varphi}-m g h \dot{\varphi} \sin \varphi\right. \\
& \left.+\left(J_{x}+m h^{2}\right) \dot{\varphi} \ddot{\varphi}\left(\varphi, \dot{\varphi}, \delta_{w, i}, u\right)\right) .
\end{aligned}
$$

$\ddot{\varphi}\left(\varphi, \dot{\varphi}, \delta_{w, i}, u\right)$ represents an appropriate model of the roll dynamics, in this work we will employ the linear model (2) connected to actuator model (3) and (5) for this purpose. To avoid the need for additional sensory equipment for roll rate and roll angle determination ${ }^{2}$, we also utilize the sampled updates of this model to generate the required trajectory of $(\varphi, \dot{\varphi})$. Due to stable dynamics, the model error is bounded.

Now, a rollover preventing constraint can be formulated by limiting the (predicted) rollover threat index to a defined critical value $\Delta_{\text {crit }}$ :

$$
C\left(\varphi, \dot{\varphi}, \delta_{w, i}, u\right):=\Delta_{\text {pred }}\left(\varphi, \dot{\varphi}, \delta_{w, i}, u\right)-\Delta_{\text {crit }} \leq 0 .
$$

To be incorporated into an allocation algorithm on the basis of [8], this inequality constraint has to be transformed into

\footnotetext{
${ }^{2}$ Note that in the employed allocation algorithm, the terms depending on the control input are the ones most decisive for the allocation's behavior. Hence, precise knowledge of roll angle and roll rate, obtained e.g. through direct measurements, would only provide a small advantage.
} 
an equivalent equality constraint $C_{e}\left(\varphi, \dot{\varphi}, \delta_{w, i}, u\right)=0$. Since it might not always be possible to prevent a violation of the constraint (which not necessarily has to be associated with an actual rollover event) it is important that $C_{e}\left(\varphi, \dot{\varphi}, \delta_{w, i}, u\right)$ is defined and finite in a region around the origin. An exponential barrier-like function of the form

$$
C_{e}\left(\varphi, \dot{\varphi}, \delta_{w, i}, u\right):=c_{1}\left(\mathrm{e}^{c_{2} C\left(\varphi, \dot{\varphi}, \delta_{w, i}, u\right)}-\mathrm{e}^{-c_{2} \Delta_{\text {crit }}}\right),
$$

where $c_{1}, c_{2}>0$, meets this requirements. Since $\Delta_{\text {pred }}\left(\varphi, \dot{\varphi}, \delta_{w, i}, u\right) \geq 0$, the second term in (13) is introduced to make $C_{e}\left(\varphi, \dot{\varphi}, \delta_{w, i}, u\right)=0$ feasible.

Remark 4.1: For $\dot{\varphi}=0$ and $\alpha_{i}=\delta_{w, i}=0 \forall i$ Assumption 4.1 is violated, but it is obvious that these situations are not associated with potential rollover, hence out of the algorithm's operational region. To define this operational region, rollover prevention can be excluded from the allocation whenever $C_{e}\left(\varphi, \dot{\varphi}, \delta_{w, i}, u\right)$ falls below a defined threshold $C_{e T}$.

\section{B. Control Allocation Algorithm}

Solving the hierarchical allocation problem (9) sequentially as its formulation implies, makes great demands on computational resources. In order to enable cost efficient real-time implementation, it would be very rewarding to find a problem setup approximating (9) in a single optimization step. A traditional approach to this task would be to impose a weighting parameter $w_{C} \gg 0$ on $C_{e}(t, x, u)$ and include it in (7) as a second constraint along $\tau_{c}-h(t, x, u)=0$. But for the given application, this proves not to provide a satisfying solution. If the weighted constraint is in an order of magnitude that dominates the input mapping, it is already too large to be sufficiently influenced by cost minimization. This means, the vehicle will brake unnecessarily aggressive in rollover-critical situations, having a negative effect on performance, reducing controllability by the driver and endangering vehicle stability. Note that the same problems would be raised by introducing rollover prevention in form of a barrier function in cost function (8): the barrier function would have to grow very large to dominate input mapping and thereby also dominate cost minimization.

Therefore, let us take a different approach to this problem. The basic control allocation problem (7) can be reformulated by introducing Lagrange multipliers $\lambda_{\tau} \in \mathbb{R}^{p}$ and the Lagrangian

$$
\ell_{\tau}\left(t, x, u, \lambda_{\tau}\right):=J(t, x, u)+w_{\tau}\left(\tau_{c}-h(t, x, u)\right)^{\mathrm{T}} \lambda_{\tau},
$$

where $w_{\tau}>0$ denotes a weighting parameter. Local minima of (7) satisfy the first order optimality conditions for $\ell_{\tau}$, which is utilized in [8] to design a control Lyapunov function

$$
V_{\tau}\left(t, x, u, \lambda_{\tau}\right):=\sigma V_{0}(t, x)+\frac{1}{2}\left(\frac{\partial \ell_{\tau}^{\mathrm{T}}}{\partial u} \frac{\partial \ell_{\tau}}{\partial u}+\frac{\partial \ell_{\tau}^{\mathrm{T}}}{\partial \lambda_{\tau}} \frac{\partial \ell_{\tau}}{\partial \lambda_{\tau}}\right),
$$

where $\sigma>0$, attracting the total state to the desired optimal solution of (7). $V_{0}:[0, \infty) \times \mathbb{R}^{n} \rightarrow \mathbb{R}$ represents a suitable differentiable Lyapunov function proving that the virtual controller $\tau_{c}=k(t, x)$ makes the origin uniformly globally exponentially stable. Inspired by the Lyapunov design of (15), the central thought of the presented approach is to approximate problem (9) by

$$
\min _{u, \lambda_{\tau}} \frac{1}{2}\left(\frac{\partial \ell_{\tau}^{\mathrm{T}}}{\partial u} \frac{\partial \ell_{\tau}}{\partial u}+\frac{\partial \ell_{\tau}^{\mathrm{T}}}{\partial \lambda_{\tau}} \frac{\partial \ell_{\tau}}{\partial \lambda_{\tau}}\right) \text { s.t. } C_{e}(t, x, u)=0 .
$$

This essentially means to minimize the Lyapunov function (15) under a constraint $C_{e}(t, x, u)=0$. Thus, if no constraint would be present, any feasible solution to this problem would bring this Lyapunov function to the origin, which is equivalent to solving (7). Under constraint $C_{e}(t, x, u)=$ 0 we obtain the minimum of the Lyapunov function for which this constraint holds. Note that this does not exactly reproduce problem setup (9), because apart from a deviation of reference tracking $\tau_{c}-h(t, x, u)$ from zero, also an increase of cost $J(t, x, u)$ compared to the unconstrained case (7) implies a non-zero Lyapunov function. But this can easily be addressed by design parameters emphasizing that cost increase is preferable. Due to the dynamic nature of the allocation algorithm, this is recommendable anyway in order to improve tracking of $\tau_{c}$. We will introduce such parameters directly into the dynamic update laws for $u$ and $\lambda_{\tau}$.

Since (7) and (16) both are instances of a general class of problems

$$
\min _{\tilde{u}} \mathcal{J}(t, x, \tilde{u}) \quad \text { s.t. } \quad \mathcal{C}(t, x, u)=0,
$$

where in (7) $\tilde{u}=u$ and for (16) $\tilde{u}=\left(u, \lambda_{\tau}\right)$, it is possible to extend the results of [8] to problems of the form (16).

Introducing Lagrange multipliers $\lambda_{C} \in \mathbb{R}^{s}$ and $U:=$ $\left[\begin{array}{ll}u^{\mathrm{T}} & \lambda_{\tau}^{\mathrm{T}}\end{array}\right]^{\mathrm{T}},(16)$ is reformulated by the Lagrangian

$\ell_{C}\left(t, x, U, \lambda_{C}\right):=\frac{1}{2}\left(\frac{\partial \ell_{\tau}^{\mathrm{T}}}{\partial u} \frac{\partial \ell_{\tau}}{\partial u}+\frac{\partial \ell_{\tau}^{\mathrm{T}}}{\partial \lambda_{\tau}} \frac{\partial \ell_{\tau}}{\partial \lambda_{\tau}}\right)+w_{C} C_{e}^{\mathrm{T}} \lambda_{C}$,

where $w_{C}>0$ denotes another weighting parameter. Note that the purpose of this parameter is not to enforce priority of the constraint $C_{e}(t, x, u)$ as in the weighting-approach described in the beginning of this section. Now, it is intended purely to perform adjustments in the algorithm's convergence behavior and should be chosen in respectively modest magnitudes.

The dynamic control allocation is defined in form of the Newton-like update law

$$
\begin{aligned}
{\left[\begin{array}{c}
\dot{u} \\
\dot{\lambda}_{\tau} \\
\dot{\lambda}_{C}
\end{array}\right] } & =-\gamma\left(\mathbb{H}_{C}^{\mathrm{T}} \mathbb{H}_{C}+\varepsilon I_{r+p+s}\right)^{-1} \mathbb{H}_{C} W_{C} \\
& \left(\left[\begin{array}{cc}
{\left[\mathbb{H}_{\tau} W_{\tau}\right.} & 0 \\
0 & I_{s}
\end{array}\right]\left[\begin{array}{c}
\frac{\partial \ell_{\tau}}{\partial u} \\
\frac{\partial \ell_{\tau}}{\partial \lambda_{\tau}} \\
w_{c} C_{e}
\end{array}\right]+w_{C}\left[\begin{array}{c}
\frac{\partial C_{e}^{\mathrm{T}}}{\partial u} \lambda_{C} \\
0 \\
0
\end{array}\right]\right)+\left[\begin{array}{c}
\zeta_{C} \\
\phi_{C}
\end{array}\right],
\end{aligned}
$$

where $W_{\tau}=W_{\tau}^{\mathrm{T}}>0, W_{C}=W_{C}^{\mathrm{T}}>0, \gamma=\gamma^{\mathrm{T}}>0$ and $\varepsilon \geq 0 . W_{\tau}$ is introduced in order to enable emphasizing that an increase of $\frac{\partial \ell_{\tau}}{\partial u}$ may be preferable over an increased $\frac{\partial \ell_{\tau}}{\partial \lambda_{\tau}}$ indicating deterioration of virtual control tracking. $W_{C}$ addresses $\ell_{C}$ in analogy ${ }^{3} . \mathbb{H}_{C}$ and $\mathbb{H}_{\tau}$ are defined by

$$
\begin{aligned}
\mathbb{H}_{C}(t, x, U) & :=\left[\begin{array}{cc}
\mathbb{H}_{\tau}^{\mathrm{T}} \mathbb{H}_{\tau} & w_{C} \frac{\partial C_{e}^{\mathrm{T}}}{\partial U} \\
w_{C} \frac{\partial C_{e}}{\partial U} & 0
\end{array}\right] \\
\mathbb{H}_{\tau}(t, x, u) & :=\left[\begin{array}{cc}
H^{\mathrm{T}} H & -w_{\tau} \frac{\partial h^{\mathrm{T}}}{\partial u} \\
-w_{\tau} \frac{\partial h}{\partial u} & 0
\end{array}\right]
\end{aligned}
$$

where $H=H^{\mathrm{T}}>0$ refers to cost function (8). The

\footnotetext{
${ }^{3}$ Since it can be shown that the terms $\alpha_{C}^{\mathrm{T}} \dot{U}$ and $\beta_{C}^{\mathrm{T}} \dot{\lambda}_{C}$ are still negative definite, introduction of $W_{\tau}$ and $W_{C}$ does not corrupt the theoretical framework of $[8]$.
} 
feedforward-like terms $\zeta_{C}, \phi_{C}$ solve

$$
\alpha_{C}^{\mathrm{T}} \zeta_{C}+\beta_{C}^{\mathrm{T}} \phi_{C}+\delta_{C}=0
$$

where

$$
\left[\begin{array}{c}
\alpha_{C} \\
\beta_{C}
\end{array}\right]:=\mathbb{H}_{C}\left[\begin{array}{c}
\frac{\partial \ell_{C}}{\partial U_{C}} \\
\frac{\partial \ell_{C}}{\partial \lambda_{C}}
\end{array}\right], \quad \delta_{C}:=-w_{\tau}^{2} \frac{\partial \ell_{C}^{\mathrm{T}}}{\partial U}\left[\begin{array}{c}
\frac{\partial h^{\mathrm{T}}}{\partial u} \dot{\tau}_{c} \\
0
\end{array}\right] .
$$

The necessary partial derivatives of the Lagrangians (14) and (18) are given by

$$
\begin{array}{lll}
\frac{\partial \ell_{\tau}}{\partial u}=\frac{\partial J}{\partial u}-w_{\tau} \frac{\partial h^{\mathrm{T}}}{\partial u} \lambda_{\tau}, & \frac{\partial \ell_{\tau}}{\partial \lambda_{\tau}}=w_{\tau}\left(\tau_{c}-h\right) \\
\frac{\partial \ell_{C}}{\partial U}=\mathbb{H}_{\tau}\left[\begin{array}{l}
\frac{\partial \ell_{\tau}}{\partial u} \\
\frac{\partial \ell_{\tau}}{\partial \lambda_{\tau}}
\end{array}\right]+w_{C} \frac{\partial C_{e}^{\mathrm{T}}}{\partial U} \lambda_{C}, & \frac{\partial \ell_{C}}{\partial \lambda_{C}}=w_{C} C_{e} .
\end{array}
$$

Note that in the definitions (20)-(21) of $\mathbb{H}_{C}$ and $\mathbb{H}_{\tau}, \frac{\partial^{2} \ell_{C}}{\partial U^{2}}$ is linearly approximated by $\mathbb{H}_{\tau}^{\mathrm{T}} \mathbb{H}_{\tau}$ and $\frac{\partial^{2} \ell_{\tau}}{\partial u^{2}}$ by $H^{\mathrm{T}} H$. This is done in order to enforce non-singularity and boundedness of these matrices and increase the region of stability. In a broader sense, it can be interpreted as trading performance for robustness. Note that in case $\mathbb{H}_{C}$ and $\mathbb{H}_{\tau}$ would be non-singular without consideration of these approximations, this performance decrease will only be minor, due to the indefinite terms in $\frac{\partial^{2} \ell_{C}}{\partial U^{2}}$ and $\frac{\partial^{2} \ell_{\tau}}{\partial u^{2}}$ being anyway small. From a practical point of view, this further provides the advantage to significantly improve computational efficiency. However, even without these approximations the algorithm is sufficiently fast to be implemented in real-time.

For a more in-depth discussion of this algorithm including a formal proof of existence, uniform boundedness and uniform global exponential convergence to a limiting optimal set, refer to [1].

\section{SIMULATION RESULTS}

The presented control scheme was tested in a simulation environment based on Daimler's proprietary multi-body simulator CASCaDE (Computer Aided Simulation of Car, Driver and Environment) for MATLAB under consideration of a passenger car. Such a vehicle is typically not prone to rollover, therefore it is burdened with a rollover promoting load of four passengers, $100 \mathrm{~kg}$ roof load and $100 \mathrm{~kg}$ trunk load. While this has a significant impact on the vehicle's dynamics, the control algorithm still considers a physical parameterization related to a nominal load of two passengers and no cargo. This proves a significant robustness of the control algorithm towards vehicle parameterization and is important to have in mind while evaluating the simulation results. Non-physical parameters of the control allocation are given in Table III. Note that critical rollover threat index $\Delta_{\text {crit }}$ is the decisive parameter for balancing yaw stabilization and rollover prevention. The sampling time is $0.02 \mathrm{~s}$.

Fig. 4 shows the simulation results for a scenario that triggers rollover even if a yaw stabilizing control scheme is utilized (such can easily be obtained by letting $w_{C}=0$ ). It considers high friction $\mu_{H}=1.2$ and a fishhook-maneuver with a maximum steering wheel angle of $180^{\circ}$, the initial vehicle speed is $120 \mathrm{~km} / \mathrm{h}$. The labels used to name signals in the plots are explained in Table II. The most dangerous situation in terms of rollover arises between $t=2$ and $2.5 \mathrm{~s}$, when the vehicle is turned from left to right. There, the yaw torque commanded by the allocation clearly deviates from its yaw stabilizing reference, indicating that rollover
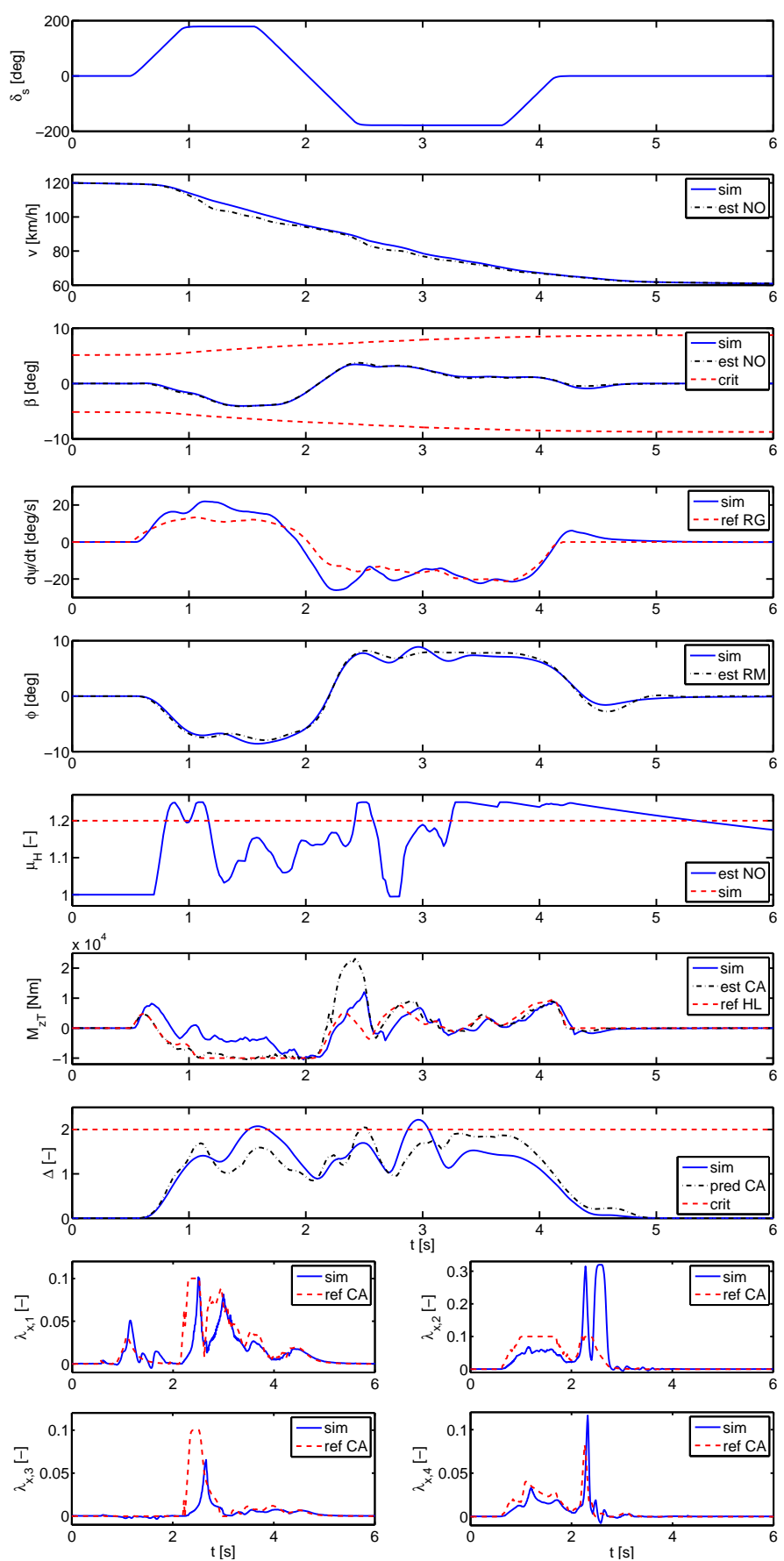

Fig. 4. Simulation results for the discussed scenario

prevention is granted priority over yaw stabilization. The desired wheel slips imply that during the rollover critical situation, braking is shifted from the outside of the turn (preventing oversteering) to the inside, creating lateral forces counteracting the roll movement. The wheel slip overshoot at $t \approx 2.5 \mathrm{~s}$ can be attributed to the small normal forces acting on the inside turn wheels. Clearly, the rollover threat index is successfully limited to the defined critical value while the vehicle's yaw motion remains stable.

In order to evaluate the performance of the developed approach, the prioritized constraint $C_{e}(t, x, u)$ has been used to track a second virtual input reference generated by a threshold-switched high-level roll controller. For this 


\begin{tabular}{|l|l|}
\hline Label & Description \\
\hline sim & Simulation model of the vehicle \\
crit & Critical value for vehicle safety \\
ref RG & Reference provided by reference generator \\
ref HL & Virtual control commanded by high-level control \\
ref CA & Desired wheel slip $\lambda_{x, i}^{\text {des } \text { provided by the allocation }}$ \\
est NO & Estimate generated by nonlinear observer \\
est RM & Estimate based on roll model \\
est CA & Estimate considering desired wheel slip $\lambda_{x, i}^{\text {des }}$ \\
pred CA & Predicted rollover threat index $\Delta_{\text {pred }}$ considering $\lambda_{x, i}^{\text {des }}$ \\
\hline
\end{tabular}

TABLE II

SIGNAL LABELS

\begin{tabular}{|ll|}
\hline$W_{\tau}$ & $\operatorname{diag}(1,1,1,1,16)$ \\
$W_{C}$ & $\operatorname{diag}\left(1,1,1,1,1,1.6 \cdot 10^{5}\right)$ \\
$\gamma$ & $\operatorname{diag}\left(5,5,5,5,10^{-3}, 1\right)$ \\
$\varepsilon$ & $10^{-9}$ \\
$w_{\tau}$ & 6 \\
$w_{C}$ & $5 \cdot 10^{2}$ \\
$H$ & $\operatorname{diag}(8,8,16,16) \cdot 10^{2}$ \\
$w_{u}$ & $10^{-4}$ \\
$u_{\min }$ & $-10^{-4}$ \\
$u_{\max }$ & $0.1+10^{-4}$ \\
$C_{e T}$ & 0.05 \\
$\Delta_{\text {crit }}$ & 2 \\
$T_{p}$ & $0.02 \mathrm{~s}$ \\
$c_{1}$ & 1 \\
$c_{2}$ & 1.5 \\
\hline
\end{tabular}

TABLE III

CONTROL ALLOCATION PARAMETERS

purpose, a Linear Quadratic Regulator based on the roll model (2) was employed and activated, whenever the estimated rollover threat index exceeds a defined threshold $\Delta_{T}=1.75$. The performance in terms of both yaw and roll stabilization was clearly deteriorated compared to the proposed consideration of rollover prevention in form of a constraint, wheel lift-off not prevented in the described simulation scenario. A detailed discussion of these results can be found in [1].

\section{CONCLUDING REMARKS}

This work presented a new approach to integrate yaw stabilization and rollover prevention of an automotive vehicle. Instead of designing controllers for both of these tasks, rollover prevention was introduced in form of a nonlinear constraint on the control allocation of the yaw stabilizing controller. The resulting algorithm was tested in a wellvalidated multi-body vehicle simulator and maintained vehicle stability in situations where a yaw stabilizing scheme fails to prevent rollover. Due to the improved integration of yaw stabilization and rollover prevention, performance was clearly increased compared to utilization of a second highlevel controller for the roll motion.

The focus of this work was on development of a suitable control allocation algorithm able to consider rollover prevention in form of a constraint, therefore the presented strategy to generate predicted values of the rollover threat index has been kept simple. Although this is already able to generate satisfying results, the potential of this aspect has hardly been exhausted and should be addressed by further research. Differences between predicted and actual rollover threat index are mainly related to inaccuracies of the roll model and differences between commanded and actual wheel slip. Hence, not least due to the utilization to generate trajectories of $(\varphi, \dot{\varphi})$, more sophisticated models of the roll dynamics should be investigated in order to replace the simple linear model. Depending on available sensory equipment, measurements or observers for roll angle and roll rate can be considered. Also ideas from [3] can be incorporated. Further, more attention should be paid to the obvious affinity to aspects of Model Predictive Control [6].

In order to improve wheel slip tracking, more advanced wheel slip controllers can be considered. In this context it should also be taken into account that common ABS are limited to low wheel slip. Since in contrast to lateral stabilization, rollover prevention has to minimize lateral forces, the ability to operate with high slip could be very rewarding.

\section{ACKNOWLEDGMENTS}

The authors are grateful to Håvard F. Grip for insightful comments and Daimler AG for access to the CASCaDE simulator through STREP project CEmACS (Complex Embedded Automotive Control Systems). This work is sponsored by the Research Council of Norway through the Strategic University Program on Computational Methods in Nonlinear Motion Control.

\section{REFERENCES}

[1] M. B. Alberding. Nonlinear Hierarchical Control Allocation for Vehicle Yaw Stabilization and Rollover Prevention. Diploma thesis, Universität Stuttgart, 2008.

[2] M. Burckhardt. Fahrzeugmechanik: Radschlupf-Regelsysteme. Vogel Fachbuch, 1993.

[3] B. C. Chen and H. Peng. A real-time rollover threat index for sports utility vehicles. In Proc. American Control Conference, San Diego, 1999.

[4] B. C. Chen and H. Peng. Rollover prevention for sports utility vehicles with human-in-the-loop evaluations. In Proc. 5th Int'l Symposium on Advanced Vehicle Control, Ann Arbor, 2000.

[5] E. Dahlberg. Commercial Vehicle Stability - Focusing on Rollover. $\mathrm{PhD}$ thesis, Royal Institute of Technology, Stockholm, 2001.

[6] P. Falcone, H. E. Tseng, F. Borrelli, J. Asgari, and D. Hrovat. MPC-based yaw and lateral stabilization via active front steering and braking. Vehicle System Dynamics, 46:611-628, 2008.

[7] H. F. Grip, L. Imsland, T. A. Johansen, T. I. Fossen, J. C. Kalkkuhl, and A. Suissa. Nonlinear vehicle side-slip estimation with friction adaptation. Automatica, 44(3):611-622, 2008.

[8] T. A. Johansen. Optimizing nonlinear control allocation. In Proc. IEEE Conference on Decision and Control, Bahamas, 2004.

[9] B. Johansson and M. Gräfvert. Untripped SUV rollover detection and prevention. In Proc. IEEE Conference on Decision and Control, Bahamas, 2004.

[10] U. Kiencke and L. Nielsen. Automotive Control Systems. Springer, 2000.

[11] D. Odenthal, T. Bünte, and J. Ackermann. Nonlinear steering and braking control for vehicle rollover avoidance. In Proc. European Control Conference, Karlsruhe, 1999.

[12] B. Schofield. Model-Based Vehicle Dynamics Control for Active Safety. PhD thesis, Lund University, 2008.

[13] B. Schofield, T. Hägglund, and A. Rantzer. Vehicle dynamics control and controller allocation for rollover prevention. In Proc. IEEE International Conference on Control Applications, Munich, 2006.

[14] J. Tjønnås and T. A. Johansen. Adaptive optimizing dynamic control allocation algorithm for yaw stabilization of an automotive vehicle using brakes. In Proc. Mediterranean Conference on Control and Automation, Ancona, 2006.

[15] J. Tjønnås and T. A. Johansen. Optimizing nonlinear adaptive control allocation with actuator dynamics. In Proc. IEEE Conference on Decision and Control, New Orleans, 2007.

[16] J. Tjønnås and T. A. Johansen. Adaptive control allocation. Automatica, 44(11):2754-2765, 2008.

[17] J. Tjønnås and T. A. Johansen. Stabilization of automotive vehicles using active steering and adaptive brake control allocations. IEEE Transactions on Control Systems Technology, 2009.

[18] P. Tøndel and T. A. Johansen. Control allocation for yaw stabilization in automotive vehicles using multiparametric nonlinear programming. In Proc. American Control Conference, Portland, 2005. 